\title{
Off-Equilibrium Dynamics at Very Low Temperatures in 3d Spin Glasses
}

\author{
Enzo Marinari ${ }^{(a)}$, Giorgio Parisi ${ }^{(b)}$, \\ Federico Ricci-Tersenghi $^{(c)}$ and Juan J. Ruiz-Lorenzo ${ }^{(d)}$ \\ (a) Dipartimento di Fisica and INFN, Università di Cagliari \\ Cittadella Universitaria, S. P. Montserrato-Sestu Km 0.709042 Montserrato (CA) (Italy) \\ Enzo.Marinari@ca.infn.it \\ (b) Dipartimento di Fisica and INFN, Università di Roma La Sapienza \\ P. A. Moro 2, 00185 Roma (Italy) \\ Giorgio.Parisi@roma1.infn. it \\ (c) Abdus Salam International Center for Theoretical Physics \\ Strada Costiera 11, P. O. Box 586, 34100 Trieste (Italy) \\ Federico.Ricci@ictp.trieste.it \\ (d) Departamento de Física Teórica I, Universidad Complutense de Madrid \\ Ciudad Universitaria, 28040 Madrid (Spain) \\ ruiz@lattice.fis.ucm.es
}

October 11, 1999

\begin{abstract}
We present a high statistic systematic study of the overlap correlation function well below the critical temperature in the three dimensional Gaussian spin glass. The offequilibrium correlation function has been studied confirming the power law behavior for the dynamical correlation length. In particular we have computed the dynamical critical exponent $z$ in a wide range of temperatures, $0.35 \leq T \leq 0.9$, obtaining a dependence $z(T)=6.2 / T$ in a very good agreement with recent experiments. Moreover, we report a study of the violation of the fluctuation-dissipation theorem for very low temperatures $T=0.5$ and $T=0.35$. All our numerical results avoid a droplet model interpretation even when $T$ is as low as $T=0.35$.
\end{abstract}




\section{Introduction}

The nature of the low temperature phase of finite dimensional spin glasses is still a subject of controversy [1, 2, 3, 4, 5].

Recently Bray, Moore, Bokil and Drossel [5, 6] have questioned many of the numerical results obtained with Monte Carlo methods in the three dimensional Edwards-Anderson (EA) model 迆, 7, 8, 9, 10.

Inspired by the study of the Migdal-Kadanoff approximation (MKA) of the EA model, they argued that the numerical results, that were obtained at temperatures $T \geq 3 / 4 T_{c}$, could be strongly affected by finite size effects and that one should go to sizes larger than the crossover length $L^{*}$ in order to see the right (droplet) behavior. They found (in the framework of the MKA) that the crossover length is $L^{*} \simeq 100$ for $T \simeq 0.7 T_{c}$ and that it decreases for lower temperatures: $L^{*} \leq 10$ when $T \leq 0.5 T_{c}$ [5], 6] (see also the comment [11]).

This is maybe the main motivation that pushed us to study the EA model in the very low temperature region: verify whether the behavior already found at $T \simeq 0.75 T_{c}$ persists at $T \leq 0.5 T_{c}$. In fact at these temperatures we can simulate (using off-equilibrium techniques) a system of size larger than $L^{*}$ (in this paper, we will present data for sizes $L=24$ and $L=64)$.

The numerical data showed in this paper have been measured in the off-equilibrium dynamical regime. This way of probing the system properties, apart from being much more similar to the experimental procedure, does not present the thermalization problems of a simulation performed at the equilibrium, that would be insurmountable obstacles at so low temperatures. The efficiency of this way of measuring has been largely tested in the recent past [12, 13, 14, 15, 16. Moreover the off-equilibrium dynamics of spin glasses has received in the last years a great attention both from the experimental [17, 18] and from the analytical 19 points of view.

Taking the measurements in the off-equilibrium regime we are able to confront the droplet model (DM) [1] and the Mean Field like theory (MF) [4] on two grounds: the off-equilibrium regime itself and the equilibrium one, that can be obtained in the limit of very large times. We can take this limit quite safely thanks to the very large time reached in our simulations.

A preliminary analysis based on the data at temperatures $T=0.7$ and $T=0.35$ was reported in reference [12. In this paper we present an extended analysis based on nine different temperatures obtaining a precise temperature dependence of the dynamical critical exponent in order to have an accurate confront with recent experiments.

\section{The model and the numerical method}

We have simulated the Gaussian Ising spin glass on a three-dimensional cubic lattice of volume $L^{3}$ with periodic boundary conditions. The Hamiltonian of the system is

$$
\mathcal{H}=-\sum_{<i j>} \sigma_{i} J_{i j} \sigma_{j}
$$

We denote by $\langle i j\rangle$ the sum over nearest neighbor pairs. $J_{i j}$ are Gaussian variables with zero mean and unit variance. 
We focus our attention on the study of the point-point overlap correlation function computed at distance $x$ and time $t$

$$
G(x, t)=\frac{1}{L^{3}} \sum_{i} \overline{\left\langle\sigma_{i+x} \tau_{i+x} \sigma_{i} \tau_{i}\right\rangle_{t}}
$$

where $\sigma$ and $\tau$ are two real replicas (systems which evolve with the same disorder) and the index $i$ runs over all the points of the lattice. As usual we denote by $\overline{(\cdots)}$ the average over the disorder and, in this context, $\langle(\cdots)\rangle_{t}$ is the average over the dynamical process until time $t$ (for a given realization of the disorder). The two replicas $(\sigma$ and $\tau$ ) evolve with different random numbers.

The simulation has been performed in a similar way to the experimental procedure: the system is prepared in a high temperature configuration (actually the initial configurations were chosen at random, i.e. $T=\infty$ ) and suddenly it is quenched below the (estimated) critical temperature, $T_{c}=0.95(3)$ 8]. Immediately we start taking the measurements, which obviously depend on time. The equilibrium behavior is recovered in the large time limit. We have used as dynamical process the standard Metropolis method.

We have simulated 4 samples ( 8 systems) of an $L=64$ lattice, measuring the correlation function at times $t=100 \cdot 2^{k}$ (with $k=0, \ldots, 13$ ) and temperatures $T=0.9,0.8,0.7$, $0.6,0.5,0.4$, and 0.35 . In addition, we have simulated 4096 samples of an $L=24$ lattice measuring at times $t=2^{k}$ (with $k=7, \ldots, 19$ ) and at three temperatures: $T=0.7,0.5$ and 0.35 .

For the study of the fluctuation-dissipation relation we have used $L=64$ systems and we have simulated them for more than $10^{7}$ Monte Carlo steps. All the simulations have been performed with the help of the parallel computer APE100 [20].

\section{Results on the correlation function}

First, we analyze the correlation functions computed with the $L=64$ runs. The study of the numerical data suggest us the following Ansatz for the time and spatial dependences of the correlation function [12]

$$
G(x, t)=\frac{\text { const }}{x^{\alpha}} \exp \left[-\left(\frac{x}{\xi(t)}\right)^{\delta}\right]
$$

where $\xi(t)$ is the dynamical correlation length. The numerical data clearly show that the dynamical correlation length depends on the time following a power law $\xi(t)=B t^{1 / z}$ where $z$ is the dynamical critical exponent. The exponents $\alpha, \delta$ and $z$ and the amplitude $B$ could, in principle, depend on the temperature. However we obtain (see below) that $\alpha, \delta$ and $B$ are almost temperature independent, while $z(T)$ is inversely proportional to $T$.

In Table 1 we report the results of our fits (always done using the CERN routine MINUIT [21]). We remark that, for a given temperature, we have fitted our numerical data to the Ansatz of Eq.(3) in two steps. In the first step we fix the distance in the correlation function and we perform the following three parameters fit in the variable $t$

$$
\log G(x, t)=A(x)-B(x) t^{-\delta / z} .
$$




\begin{tabular}{|c|c|c|c|c|}
\hline$T$ & $z(T)$ & $\delta$ & $B^{-\delta}$ & $\alpha$ \\
\hline \hline 0.9 & $6.85(1.0)$ & $1.37(11)$ & $1.02(4)$ & $0.60(7)$ \\
\hline 0.8 & $7.5(1.3)$ & $1.37(9)$ & $1.06(4)$ & $0.49(9)$ \\
\hline 0.7 & $9.3(0.7)$ & $1.50(5)$ & $1.02(4)$ & $0.53(5)$ \\
\hline 0.6 & $10.3(1.2)$ & $1.38(3)$ & $1.09(4)$ & $0.49(16)$ \\
\hline 0.5 & $11.7(1.8)$ & $1.43(2)$ & $1.04(4)$ & $0.59(20)$ \\
\hline 0.4 & $14.1(2.4)$ & $1.45(4)$ & $0.99(3)$ & $0.60(26)$ \\
\hline 0.35 & $19.9(3.8)$ & $1.41(6)$ & $1.03(7)$ & $0.29(32)$ \\
\hline
\end{tabular}

Table 1: Parameters of the point-point correlation function

We have found that $\delta / z$ is independent of $x$. The second step has been to extract from $A(x)$ and $B(x)$ the exponents $\alpha$ and $\delta$ and the amplitude $B$ using the formulæ: $A(x)=$ const $-\alpha \log x$ and $B(x)=B^{-\delta} x^{\delta}$. We report our final values of $z, \delta, B$ and $\alpha$ in Table $[$.

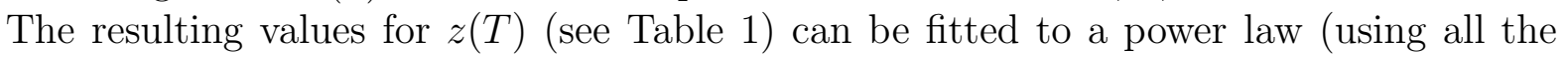
temperatures of Table 1) obtaining

$$
z(T)=6.4(6) T^{-0.96(20)} .
$$

From the previous fit we can guess a simpler law for the dynamical critical exponent $z(T)=$ $a / T$, obtainingt

$$
z(T)=\frac{6 \cdot 2(3)}{T} .
$$

This kind of behavior suggests that the low temperature dynamics in spin glasses is dominated mainly by activated processes with free energy barriers diverging logarithmically with the size of the system.

We can finally write down the dependence of the dynamical correlation length on the time as well as on the temperature:

$$
\xi(t, T) \propto t^{T / 6.2(3)}=t^{0.161(8) T}=t^{0.153(12) T / T_{c}},
$$

where we have assumed that the temperature of the phase transition is $T_{c}=0.95(3)$ [ 8 ]. The agreement of the previous formula with the experiments is very good. We recall that in experiments [17] it was found the following dependence for the dynamical correlation length

$$
\xi(t, T) \propto t^{0.170 T / T_{g}} .
$$

where $T_{g}$ is the experimental critical temperature (the authors of this result do not quote the error in the exponent).

A further check of Eq.(3) would be the collapse of the data (measured at different times and different temperatures) when plotting $G(x, t) x^{\alpha}$ versus $x / t^{1 / z(T)}$. To this purpose we use the data from 4 samples of the $64^{3}$ runs, together with those measured on 4096 samples of $24^{3}$ runs. We remark that, in the $24^{3}$ runs, the volume is near 19 times less than the $L=64$ runs but we have computed 1000 times more samples and so we expect the errors to be smaller.

\footnotetext{
${ }^{1}$ This law was found by Kisker et al. for the \pm 1 three dimensional spin glass (See references [22, 23]). Moreover this law was guessed for the Gaussian model using numerical data taken at temperatures $T=0.7$ and $T=0.35$ in reference [12].
} 

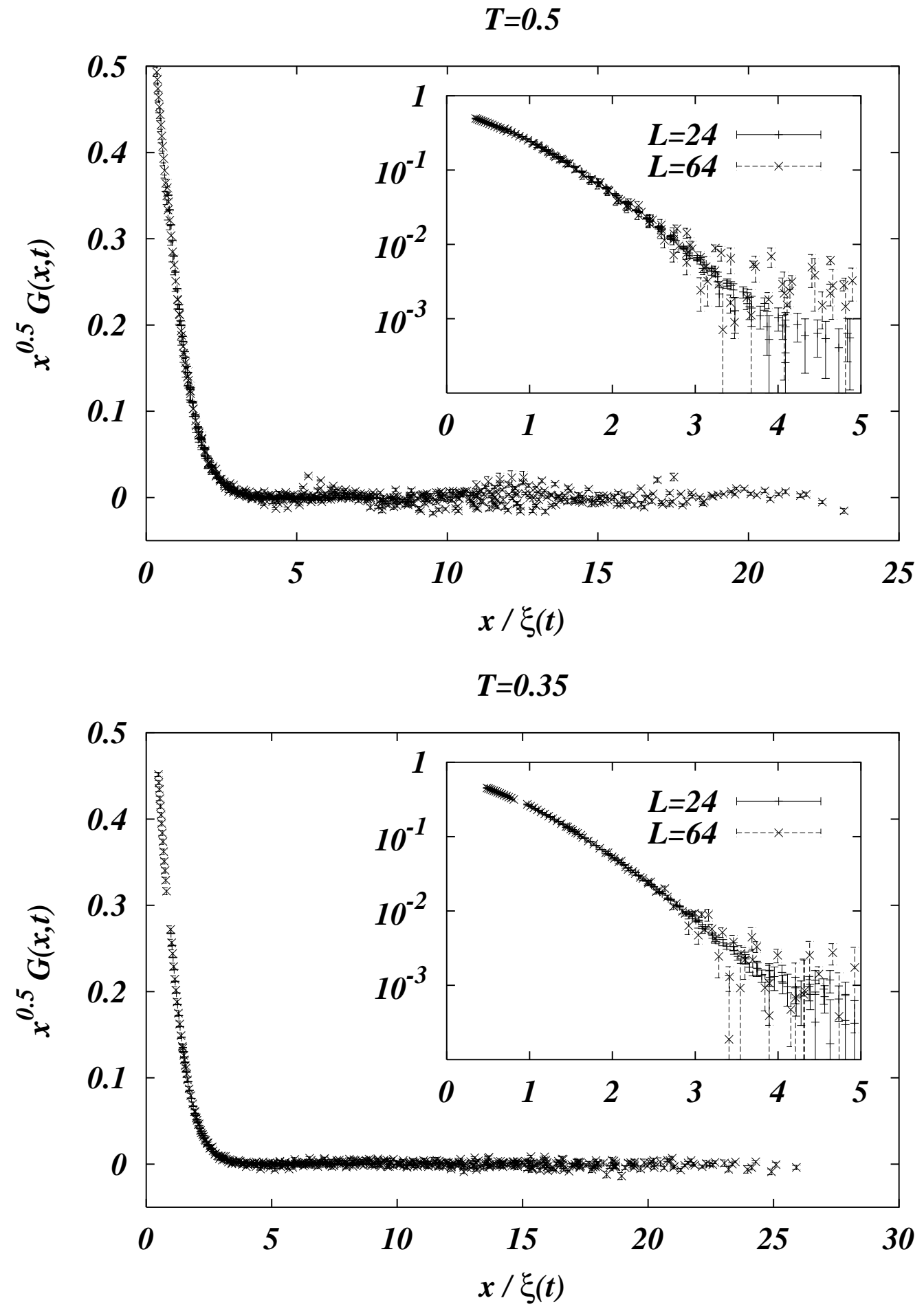

Figure 1: Scaling plot for the correlation function $G(x, t)$ measured at two very low temperatures, $T=0.5$ and 0.35 , and two lattice sizes, $L=24$ and 64 . It shows that the finite size effects are negligible and it also gives reliability to our estimate for $\xi(t)$. 


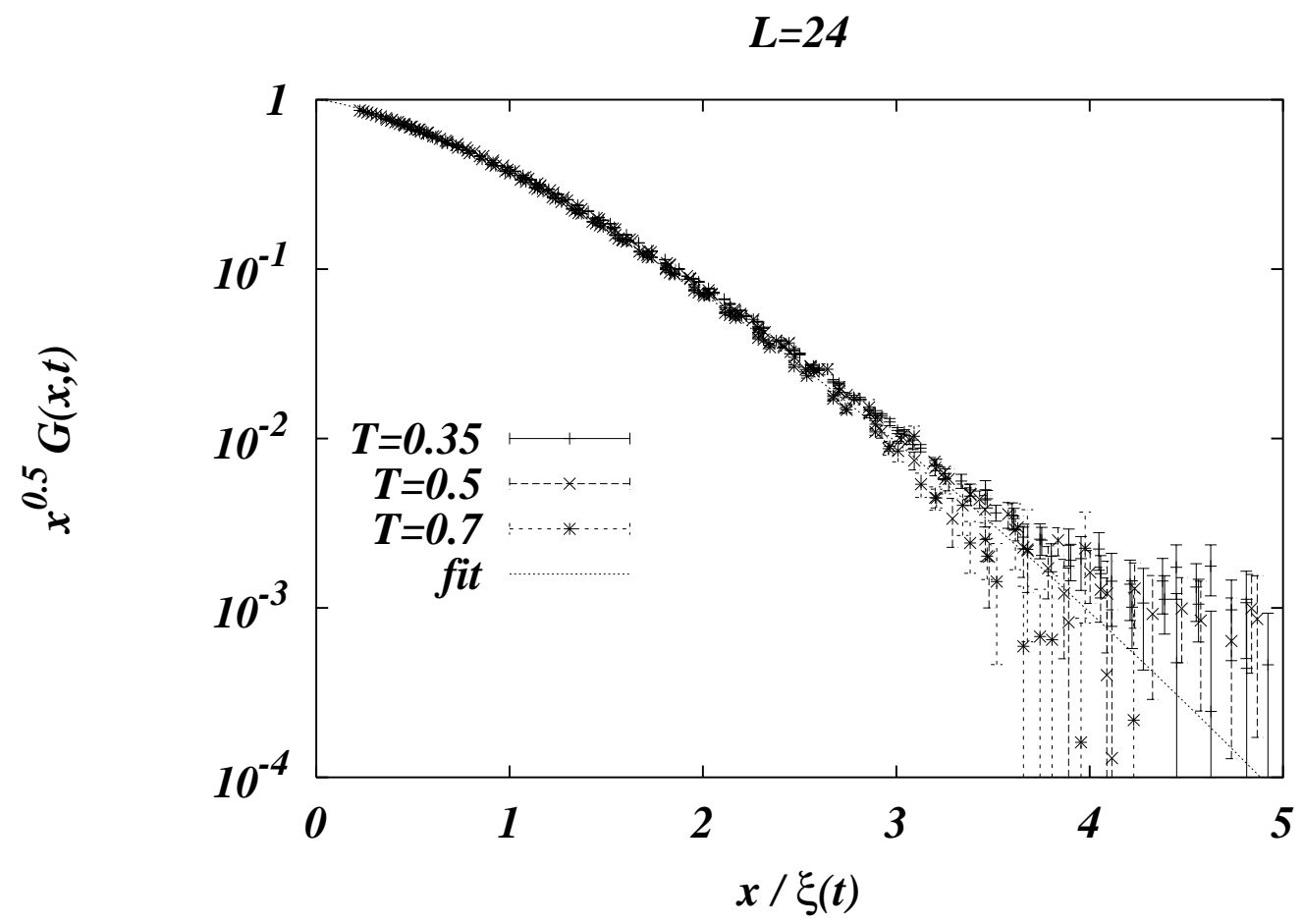

Figure 2: The scaled data for $G(x, t)$ are well described by a temperature-independent scaling function, that can be very well approximated by our fit.

In Fig. 1 we plot the correlation function for two low temperatures $(T=0.5$ and 0.35$)$ using as variables $x / \xi(t)$ and $x^{\alpha} G(x, t)$ (we have taken $\alpha=0.5$, see Table 1). In the plots we use the data from both runs $(L=24$ and $L=64)$ and they superimpose perfectly. In the insets we present the same data in a log-linear scale in order to let the reader evaluate better the collapse. It is clear that the scaling is impressive even at the lowest temperature $T=0.35$. We can also state that the finite size effects are negligible for the lattice sizes used.

Scaling arguments tell us that the more general scaling function for the correlation function is (for large $x$ and $t$ )

$$
G(x, t) \propto x^{-\alpha} \mathcal{G}\left(\frac{x}{\xi(t)}\right),
$$

where the scaling function $\mathcal{G}(y)$ is smooth. Moreover, in the scaling regime, $\mathcal{G}(y)$ should not depend neither on temperature nor on the lattice size. Note that in our Ansatz (3) we have chosen an exponential function for the scaling function: $\mathcal{G}(y) \propto \exp \left(-y^{\delta}\right)$, and we show that it fits very well the data. However to check that our estimates of $\alpha$ and $\xi(t)$ are correct we do not need to know $\mathcal{G}(y)$. We can simply plot $x^{\alpha} G(x, t)$ versus $x / \xi(t)$ (as it was done in Fig. 1) and check how well the data collapse.

In order to check the temperature independence of $\mathcal{G}(y)$ we show in Fig. 2 the scaling function for three different temperatures $(T=0.35,0.5$ and 0.7$)$, together with the exponential function $\exp \left[-y^{1.42(2)}\right]$ (see Table 1) obtained through the fitting procedure. It is 
clear that the scaling function is really temperature-independent and it can be very well approximated by the exponential function as we have chosen in our Ansatz.

Another interesting issue is the extrapolation of the correlation function to infinite time. In this limit we can compare again our numerical results with the predictions of the droplet model and with that of the RSB theory. In the former the extrapolated correlation function tends to the value $q_{\mathrm{EA}}^{2}$ for large distances, whereas the RSB prediction is a pure power law going asymptotically to zero [24]. Our Ansatz, which describes perfectly the numerical data, supports the RSB prediction even for the lowest temperatures.

Nevertheless, we have tried to fit our data with a functional dependence compatible with the droplet model, that is, $G(x, t)=G_{\infty}(x) \mathcal{G}(x / \xi(t))$, where $G_{\infty}(x)=A x^{-\alpha}+C$. If $C=0$, then the previous formula is exactly our Ansatz (and it implies a breaking of the replica symmetry), while if $C=q_{\mathrm{EA}}^{2}$ then it would support a droplet picture. Fitting the data to the previous formula, $G_{\infty}(x)$, we have found that at every temperature and even at $T=0.35$ (our lowest temperature), the best value for $C$ is always compatible with zero. At very low temperatures, i.e. $T=0.35$, the Edwards-Anderson order parameter is so close to one $\left(q_{\mathrm{EA}} \simeq 1\right)$ than we can safely distinguish between the two competing theories. In fact in the droplet like formula we have that $G_{\infty}(x)$ is almost constant? and so we simply should fit the data into the scaling formula of Eq.(9) without the factor $x^{-\alpha}$ in order to check the correctness of the droplet model.

In Fig. 3 we present the data rescaled with the formula suggested by the droplet model (left plot) and with that implied by RSB (right plot). It is clear that the RSB prediction fits much better the numerical data. Note that the data error is sufficiently small to affirm safely that the data in the left plot have no collapse at all. A scaling plot like the left one has been recently presented by Komori et al. in [25] (see also reference [26]). We believe that the rather poor collapse of their data (see Fig. 5 in [25]) is due to the fact that they neglect the factor $x^{-\alpha}$ in the scaling formula. A much better collapse would be obtained by plotting $\sqrt{x} G(x, t)$ versus $x / \xi(t)$ (see 26]).

\section{Fluctuation dissipation relation at very low temper- atures}

Now, we present the results of the analysis based on the generalization of the fluctuationdissipation theorem (FDT) in the out of equilibrium regime [27. In this section we will focus on the scaling properties of the aging region and the violation of fluctuation-dissipation at very low temperatures.

A preliminary analysis was done in [28] studying the violation of FDT at temperature, $T=0.7$. Here we have simulated different lower temperatures and so, as byproduct, we can study the scaling properties of the violation of FDT. An analogous analysis, with many temperatures, was done in [28] but on the four-dimensional EA model.

For the sake of conciseness, we do not repeat all the formalism and we address the interested reader to one of the previous publications on the subject [27, 29, 28, 30, 15]. Here

\footnotetext{
${ }^{2}$ Actually it slowly decreases from 1 to $q_{\mathrm{EA}}^{2} \simeq 1$, but for all our purposes it can be considered as a constant.
} 


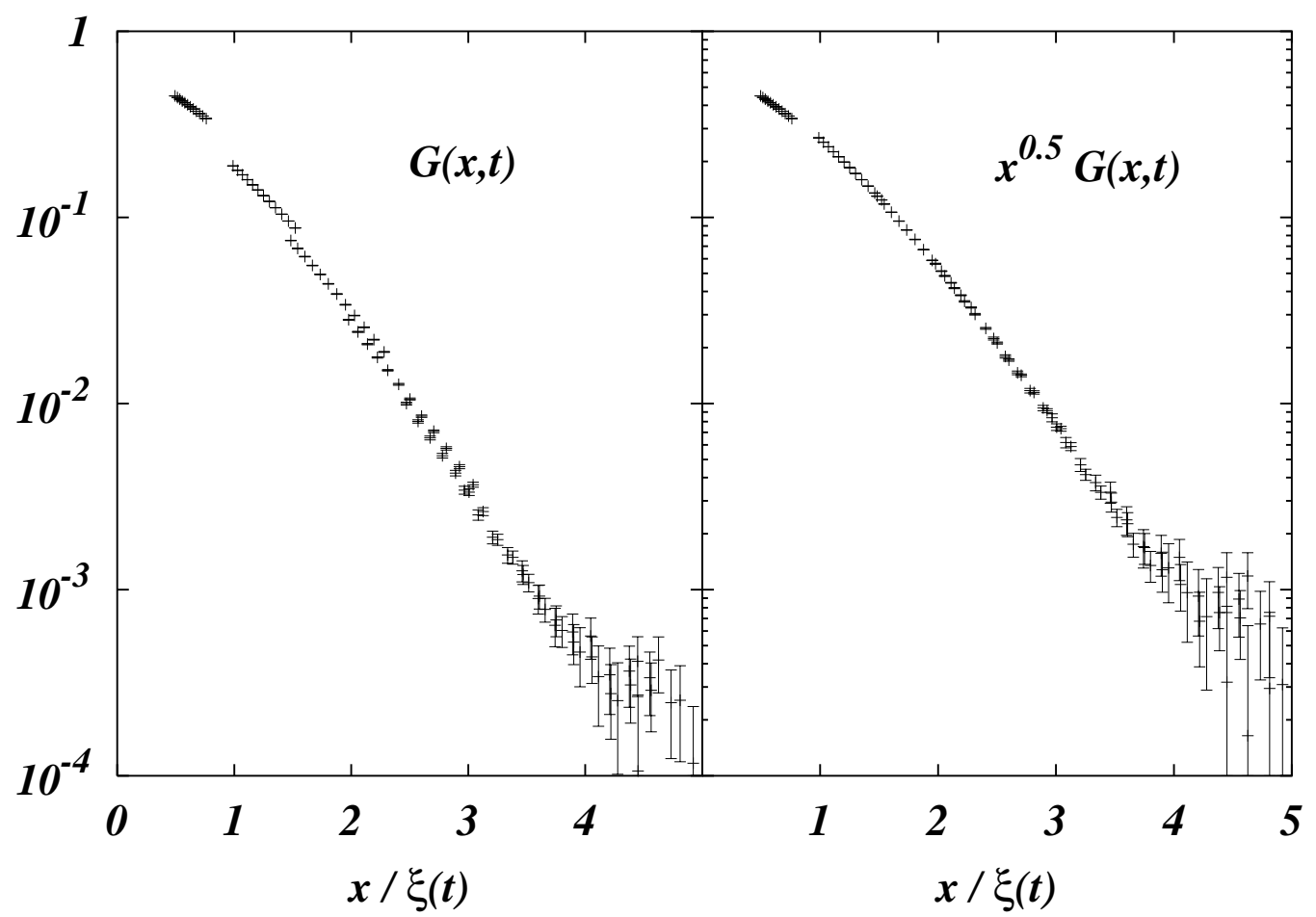

Figure 3: The comparison of these two scaling plots clearly shows that the $\sqrt{x}$ factor is essential in order to collapse the correlation function data. The temperature is $T=0.35$ and the lattice size $L=24$. 
we simply recall the main formulæ that we use. As usual we define the integrated response to a very small external field as

$$
\chi\left(t, t_{w}\right)=\lim _{h_{0} \rightarrow 0} \frac{1}{h_{0}} \int_{t_{w}}^{t} R\left(t, t^{\prime}\right) h\left(t^{\prime}\right) \mathrm{d} t^{\prime},
$$

where $h(t)=h_{0} \theta\left(t-t_{w}\right)$ and $R\left(t, t^{\prime}\right)=\frac{1}{N} \sum_{i} \frac{\partial\left\langle s_{i}(t)\right\rangle}{\partial h\left(t^{\prime}\right)}$. The autocorrelation function is defined as

$$
C\left(t, t_{w}\right)=\frac{1}{N} \sum_{i}\left\langle s_{i}(t) s_{i}\left(t_{w}\right)\right\rangle .
$$

Relating these two functions, in the large times limit, via

$$
T \chi\left(t, t_{w}\right)=S\left(C\left(t, t_{w}\right)\right)
$$

we have that, at the equilibrium, the fluctuation-dissipation theorem (FDT) holds and $S(C)=1-C$, while in the aging regime the function $S(C)$ can be linked to the equilibrium overlap distribution through $P(q)=-\left.\frac{\partial^{2} S(C)}{\partial C^{2}}\right|_{C=q}$ 28, 31.

Models that, in the frozen phase, do not show any breaking of the replica symmetry, have, at the equilibrium level, a static $P(q)=\delta\left(q-q_{\mathrm{EA}}\right)$, which dynamically corresponds to the absence of response in the aging regime. This means that, plotting $\chi\left(t, t_{w}\right)$ versus $C\left(t, t_{w}\right)$, we obtain a horizontal line in the range $C \leq q_{\mathrm{EA}}$ 30 (in the quasi-equilibrium regime, $C \geq q_{\mathrm{EA}}$, and it always holds $T \chi=1-C$ independently of the model)

In Fig. 4 we show the results for different temperatures in the usual plot $\chi\left(t, t_{w}\right)$ versus $C\left(t, t_{w}\right)$. Note that in this plot the FDT line is $\chi=(1-C) / T$ and so it is different for different temperatures. It is quite clear that, even for very large times, the curves are far from been horizontal when they leave the FDT line. This result gives more evidence in favor of a replica symmetry breaking in the very low temperature phase of the 3D EA model [28].

We present the data for different temperatures on a single plot in order to make more evident the fact that the numerical data seem to stay on the same curve once the system enters into the aging regime, i.e. when the points leave the FDT line. This kind of behavior has been observed in the four-dimensional EA model [28] and it is reminiscent of the meanfield solution.

Indeed in the SK model, using the Parisi-Toulouse (PAT) hypothesis [32], it can be shown 28] that

$$
S(C)=\left\{\begin{array}{cl}
1-C & \text { for } C \geq q_{\mathrm{EA}}(T) \\
T \sqrt{1-C} & \text { for } C \leq q_{\mathrm{EA}}(T)
\end{array} .\right.
$$

The formula can be easily generalized assuming a generic power law behavior in the aging regime: $S(C)=T A(1-C)^{B}$ (the mean-field value for the exponent is $B=1 / 2$ ).

We use this generalization to fit the data and we obtain very good results. The best fit parameters have been estimated from the collapse of the data reported in Fig. 1 and they are $A \simeq 0.7$ and $B \simeq 0.41$ (to be compared with the mean-field values $A=1$ and $B=1 / 2$, and those obtained for the $4 \mathrm{D}$ EA model $A \simeq 0.52$ and $B \simeq 0.41$ [28]).

In order to show the validity of the fitting formula, we present in Fig. 5 the collapse of the scaled data using the variables $x=(1-C) T^{-\phi}$ and $y=\chi T^{1-\phi}$, where $\phi=\frac{1}{1-B}=1.7$. It is easy to see that, if the previous scaling holds, the data should stay on two power laws: 


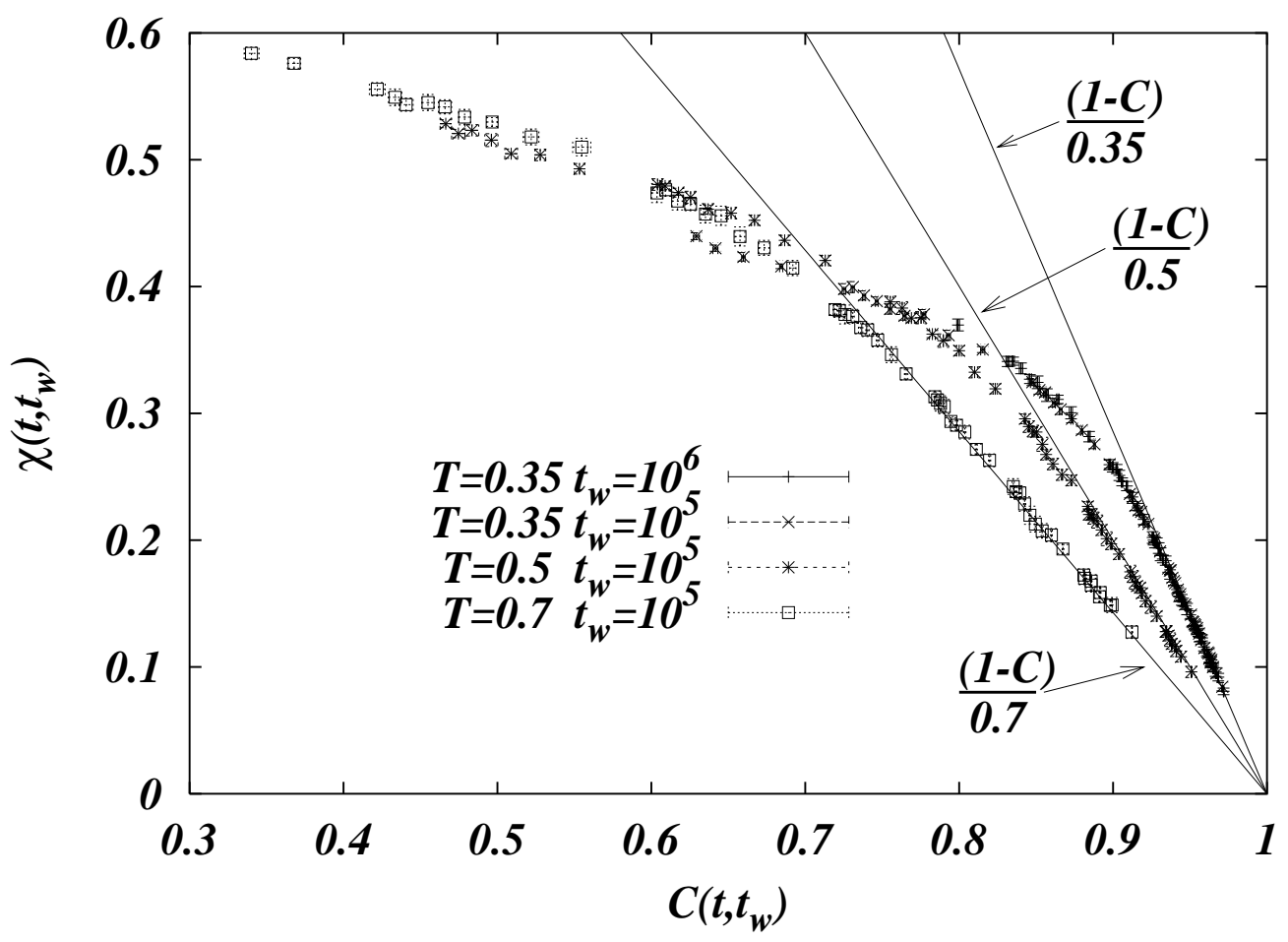

Figure 4: Response against the autocorrelation function for three different temperatures and lattice size $L=64$. Note that in this figure we plot $\chi\left(t, t_{w}\right)$ versus $C\left(t, t_{w}\right)$. The data stay on a single universal curve when they leave the FDT lines. This curve is clearly non horizontal and this hints for a breaking of the replica symmetry in the very low temperature phase of the EA model. 


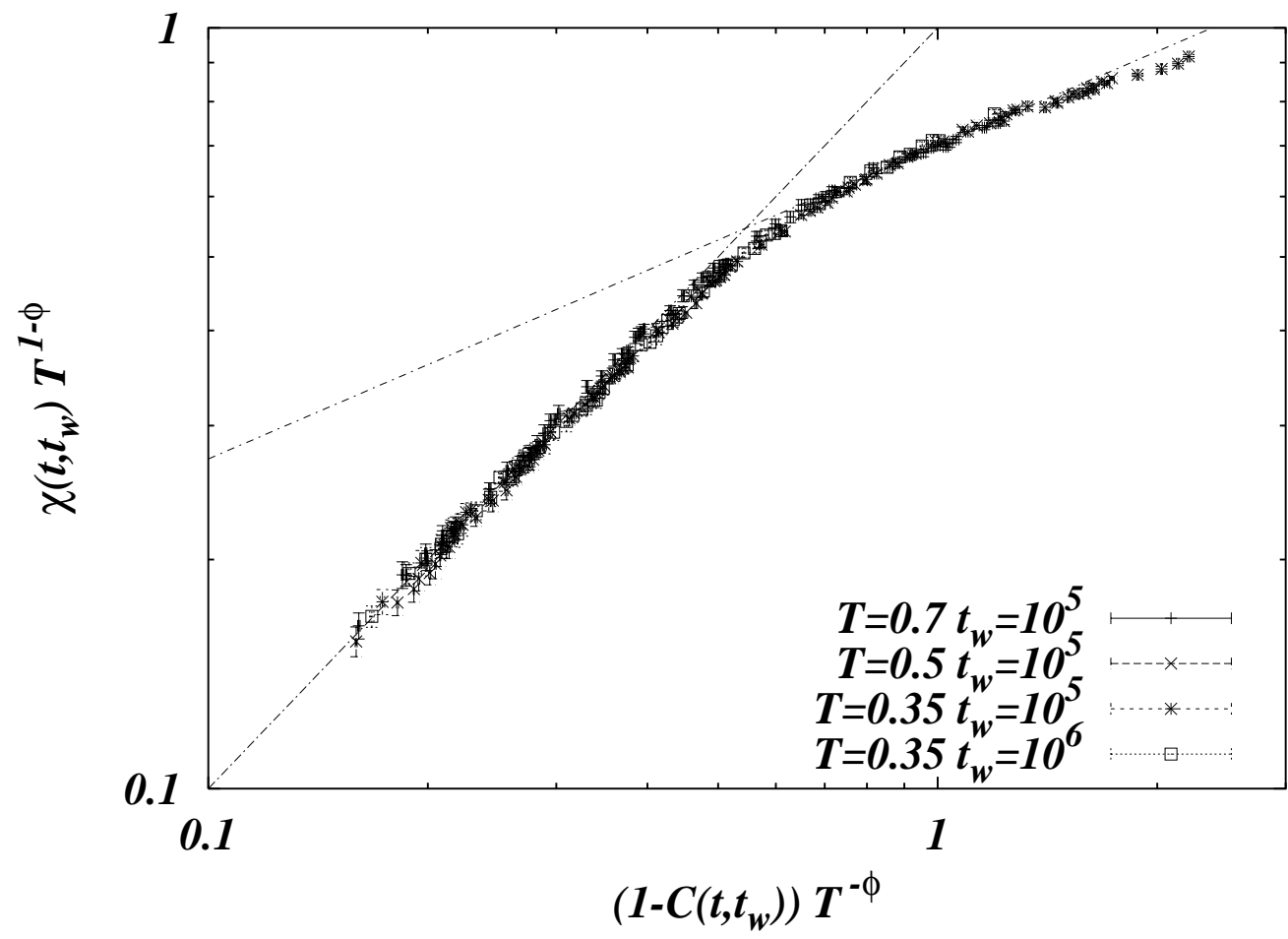

Figure 5: Scaling plot of the curves shown in the previous figure: $\chi T^{1-\phi}$ versus $(1-C) T^{-\phi}$ with $\phi=1.7$. In the FDT regime (left part of the figure) the scaled data stay on the line $y=x$, while in the off-equilibrium regime (right part of the figure) they follow the plotted power law $y=A x^{B}$ with $B=0.41$. 
$y=x$ and $y=A x^{B}$ in the quasi-equilibrium and aging regime, respectively. The two power laws are reported in Fig. 5 .

Even if we may expect a breakdown of the assumed scaling for large values of the scaling variable $x$ (i.e. the scaled data are no longer described by a power law), we note however that for a quite large range the collapse is very good and very well approximated by a power law. Moreover, we remark that the collapse has been obtained adjusting only one parameter.

\section{Discussion}

We have studied the off equilibrium dynamics of the three dimensional Gaussian spin glass in the very low temperature phase. In particular we have studied the scaling properties of the dynamical overlap correlation functions and the scaling properties of the violation of the fluctuation-dissipation.

We have tried to fit our correlation functions to the functional form predicted by the droplet model but the fits were poor. Moreover a correlation length diverging following a power law with the time implies, as was noted by Rieger [23], barriers diverging not as $L^{\psi}$ (as predicted by the droplet model with the lower bound $\psi \geq \theta \simeq 0.2$ ) but as $\log L$. This latter results implies $\psi=0$, hence violating the droplet lower bound.

It is interesting to note that the experimental data could be fitted to the droplet formula assuming that $\psi=\theta$ [17]. However while both, the results of numerical simulations and the experiments are in very good agreement with a power law fit for $\xi(t, T)$, the numerical fit assuming a droplet formula for $\xi(t, T)$ [22] disagrees with the experimental fit assuming the same hypothesis [17].

As it has been noted above, our final result for the dynamical correlation length is in a very good agreement with the experimental result.

We remark that the same scenario (power law dependence of $\xi(t, T)$ and linear dependence of $1 / z$ with temperature) also emerges in four and six dimensions. In the latter case it was found that $z(T)=4 T_{c} / T$ [14] $(z=4$ at the transition is the value predicted by Mean Field) while in the former one $z(T)=5.5 T_{c} / T$ [13]. Moreover in these two dimensions the overlap correlation function constrained to zero overlap follows a pure power law as in three dimensions.

If we send to infinity the time in our Ansatz for the overlap-overlap correlation function we obtain a pure power decay $G(x) \propto x^{-\alpha}$ with $\alpha \simeq 0.5$, with a small dependence of $\alpha$ on the temperature for the whole spin glass phase. We recall again that the droplet prediction is $G(x) \rightarrow q_{\mathrm{EA}}^{2}$ in contradiction with our numerical correlation functions (this fact was already noted in [12]). Instead, the pure power behavior is supported by the Gaussian approximation using the Mean Field solution [24, 国.

One could argue that the simulated temperatures are not low enough and the times and sizes not large enough in order to see the "true" (droplet) behavior of the EA model. However, as we stressed in the Introduction, $L=64$ is large enough for temperatures as low as $T=0.35$ and $T=0.5$. Moreover our large times extrapolations are very safe thanks to the measurements have been taken over 6 time decades.

We have shown numerical results that contradict the droplet predictions in a wide range of temperatures $(0.35 \leq T \leq 0.9)$. In particular we point out that our results (both for 
correlation functions and for violation of FDT) at a very low temperature, $T=0.35$, support a Mean Field picture.

Finally, we remark that using the (PAT) Mean Field scaling relations for the $P(q)$ [32] we have obtained a very good scaling plot of the violation of fluctuation-dissipation (like in four dimensions [28]). This provides us another strong evidence calling for a low temperature phase being well described by Mean Field 四.

\section{Acknowledgments}

JJRL is partially supported by CICyT AEN97-1693. JJRL wishes to thank L.A. Férnandez and A. Muñoz Sudupe for interesting suggestions. Moreover we wish to thank A. J. Bray for pointing out an arithmetic error in equation (7).

\section{References}

[1] W. L. McMillan, J. Phys. C 17, 3179 (1984). A. J. Bray, M. A. Moore, in Heidelberg Colloquium on Glassy Dynamics, Lecture Notes in Physics 275, J. L. van Hemmen and I. Morgenstern (ed. Springer, Berlin). D. S. Fisher, D. A. Huse, Phys. Rev. Lett. 56, 1601 (1986) and Phys. Rev. B 38386 (1988).

[2] M. Mezard, G. Parisi and M.A. Virasoro, Spin Glass Theory and Beyond. (World Scientific, Singapore, 1987).

[3] C. N. Newman and D. L. Stein, Phys. Rev. Lett. 76, 515 (1996).

[4] E. Marinari, G. Parisi, F. Ricci-Tersenghi, J. J. Ruiz-Lorenzo and F. Zuliani. cond-mat/9906076.

[5] M.A. Moore, H. Bokil and B. Drossel, Phys. Rev. Lett. 81, 4252 (1998).

[6] H. Bokil, A.J. Bray, B. Drossel, and M.A. Moore, Phys. Rev. Lett. 82, 5177 (1999).

[7] E. Marinari, G. Parisi and J.J. Ruiz-Lorenzo, "Numerical Simulations of Spin Glass Systems" in "Spin Glasses and Random Fields", edited by P. Young. World Scientific (Singapore 1997).

[8] E. Marinari, G. Parisi and J.J. Ruiz-Lorenzo, Phys. Rev. B 58, 14852 (1998).

[9] E. Marinari and F. Zuliani, cond-mat/9904303.

[10] E. Marinari, G. Parisi, F. Ricci-Tersenghi and J.J. Ruiz-Lorenzo, J. Phys. A 31, L481 (1998).

[11] E. Marinari, G. Parisi, J.J. Ruiz-Lorenzo and F. Zuliani, Phys. Rev. Lett. 82, 5176 (1999).

[12] E. Marinari, G. Parisi, J.J. Ruiz-Lorenzo and F. Ritort, Phys. Rev. Lett. 76, 843 (1996). 
[13] G. Parisi, F. Ricci-Tersenghi and J.J. Ruiz-Lorenzo, J. Phys. A 29, 7943 (1996).

[14] G. Parisi, P. Ranieri, F. Ricci-Tersenghi and J.J. Ruiz-Lorenzo, J. Phys. A 30, 7115 (1997).

[15] G. Parisi, F. Ricci-Tersenghi and J.J. Ruiz-Lorenzo, Phys. Rev. B 57, 13617 (1998).

[16] E. Marinari, G. Parisi and F. Zuliani, J. Phys. A 31, 1181 (1998).

[17] Y. G. Joh, R. Orbach, G. G. Wood, J. Hammann and E. Vincent, Phys. Rev. Lett. 82, 438 (1999).

[18] Y. G. Joh, R. Orbach and J. Hammann, Phys. Rev. Lett. 77, 4648 (1996). K. Jonason, E. Vincent, J. Hammann, J. P. Bouchaud and P. Nordblad, Phys. Rev. Lett. 81, 3243 (1998).

[19] J. -P. Bouchaud et al., in "Spin Glasses and Random Fields", edited by P. Young. World Scientific (Singapore 1997) p. 161.

[20] C. Battista et al. Int. J. High Speed Comp, 5, 637 (1993).

[21] Minuit. CERN Library. http://www.cern.ch.

[22] J. Kisker, L. Santen, M. Schreckenberg and H. Rieger, Phys. Rev. B 53, 6418 (1996).

[23] H. Rieger, in Annual Reviews of Computational Physics II (World Scientific 1995, Singapore) p. 295.

[24] C. De Dominicis, I. Kondor and T. Temesvári, in "Spin Glasses and Random Fields", edited by P. Young. World Scientific (Singapore 1997) p. 119.

[25] T. Komori, H. Yoshino and H. Takayama, preprint cond-mat/9904143.

[26] E. Marinari, G. Parisi and J. J. Ruiz-Lorenzo, "Comment on "Numerical Study on Aging Dynamics in the 3D Ising Spin Glass Model". cond-mat/9904321. Unpublished.

[27] L. Cugliandolo and J. Kurchan, Phys. Rev. Lett. 71, 173 (1993).

[28] E. Marinari, G. Parisi, F. Ricci-Tersenghi and J. J. Ruiz-Lorenzo, J. Phys. A 31, 2611 (1998).

[29] S. Franz and H. Rieger, J. Stat. Phys. 79, 749 (1995).

[30] G. Parisi, F. Ricci-Tersenghi and J. J. Ruiz-Lorenzo, Eur. Phys. Jour. B 11, 317 (1999).

[31] S. Franz, M. Mezard, G. Parisi and L. Pelliti, Phys. Rev. Lett. 81, 1758 (1998).

[32] G. Parisi and G. Toulouse, J. de Phys. Lett. 41, L361 (1980). G. Parisi, G. Toulouse and Vannimenus, J. de Phys. 42565 (1981). 